Document downloaded from:

http://hdl.handle.net/10251/180319

This paper must be cited as:

Gómez García, JF.; Trenor Gomis, BA.; Sebastián Aguilar, R. (2020). Prediction of CRT Activation Sequence by Personalization of Biventricular Models from Electroanatomical Maps. Lecture Notes in Computer Science. 12009:342-351. https://doi.org/10.1007/978-3030-39074-7_36

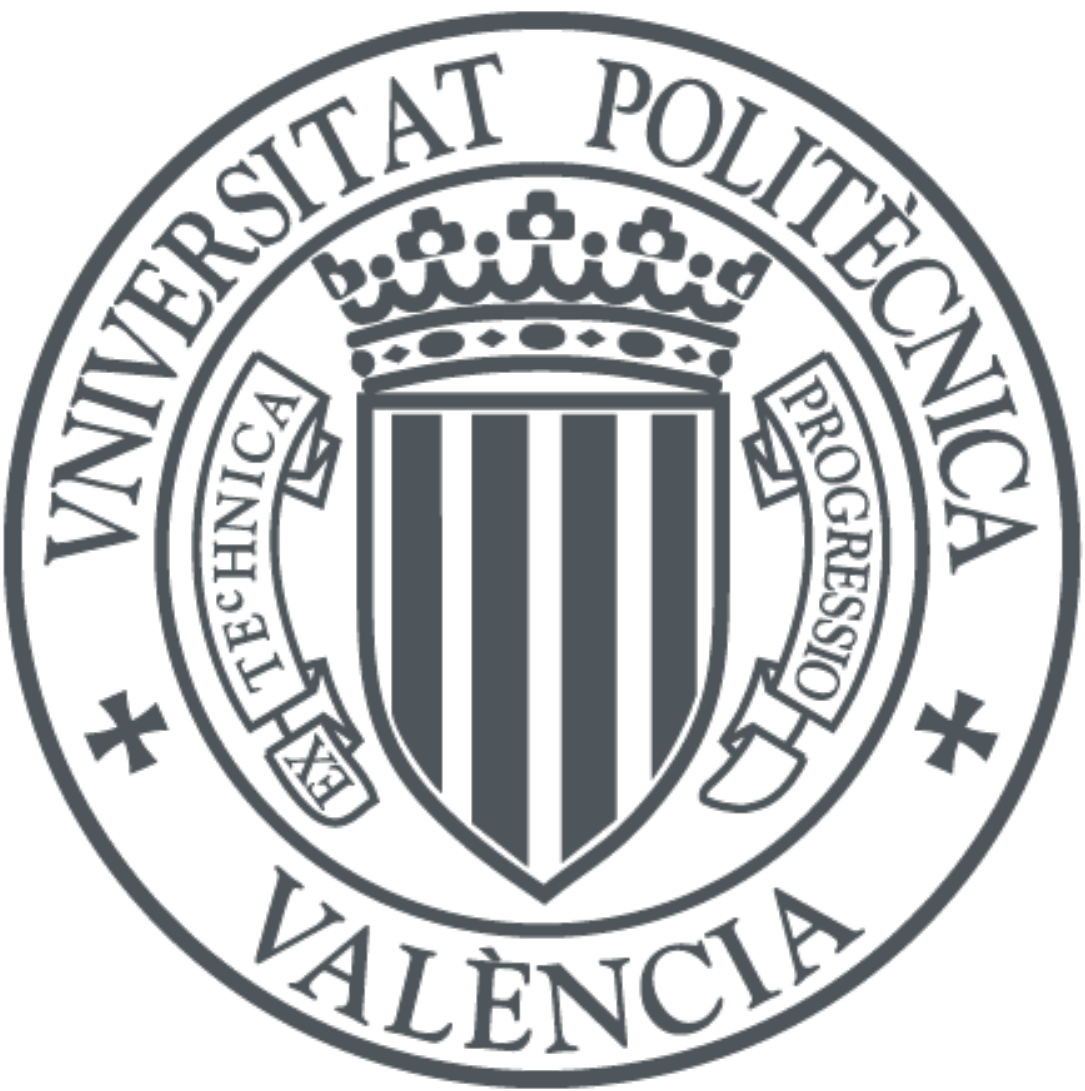

The final publication is available at

https://doi.org/10.1007/978-3-030-39074-7_36

Copyright Springer-Verlag

Additional Information 


\section{Metadata of the chapter that will be visualized in SpringerLink}

\begin{tabular}{|c|c|c|}
\hline Book Title & \multicolumn{2}{|c|}{$\begin{array}{l}\text { Statistical Atlases and Computational Models of the Heart. Multi-Sequence CMR Segmentation, CRT- } \\
\text { EPiggy and LV Full Quantification Challenges }\end{array}$} \\
\hline \multicolumn{3}{|l|}{ Series Title } \\
\hline Chapter Title & \multicolumn{2}{|c|}{$\begin{array}{l}\text { Prediction of CRT Activation Sequence by Personalization of Biventricular Models from } \\
\text { Electroanatomical Maps }\end{array}$} \\
\hline Copyright Year & \multicolumn{2}{|l|}{2020} \\
\hline Copyright HolderName & \multicolumn{2}{|c|}{ Springer Nature Switzerland AG } \\
\hline \multirow[t]{13}{*}{ Author } & Family Name & Gomez \\
\hline & Particle & \\
\hline & Given Name & Juan Francisco \\
\hline & Prefix & \\
\hline & Suffix & \\
\hline & Role & \\
\hline & Division & Centre for Research and Innovation in Bioengineering (Ci2B) \\
\hline & Organization & Universitat Politecnica de Valencia \\
\hline & Address & Valencia, Spain \\
\hline & Division & \\
\hline & Organization & International University of Valencia (VIU) \\
\hline & Address & Valencia, Spain \\
\hline & Email & \\
\hline \multirow[t]{10}{*}{ Author } & Family Name & Trenor \\
\hline & Particle & \\
\hline & Given Name & Beatriz \\
\hline & Prefix & \\
\hline & Suffix & \\
\hline & Role & \\
\hline & Division & Centre for Research and Innovation in Bioengineering (Ci2B) \\
\hline & Organization & Universitat Politecnica de Valencia \\
\hline & Address & Valencia, Spain \\
\hline & Email & \\
\hline \multirow[t]{10}{*}{ Corresponding Author } & Family Name & Sebastian \\
\hline & Particle & \\
\hline & Given Name & Rafael \\
\hline & Prefix & \\
\hline & Suffix & \\
\hline & Role & \\
\hline & Division & $\begin{array}{l}\text { Computational Multiscale Simulation Lab (CoMMLab), Department of } \\
\text { Computer Science }\end{array}$ \\
\hline & Organization & Universitat de Valencia \\
\hline & Address & Valencia, Spain \\
\hline & Email & rafael.sebastian@uv.es \\
\hline
\end{tabular}


Abstract

Optimization of lead placement and interventricular delay settings in patients under cardiac resynchronization therapy is a complex task that might benefit from prior information based on models.

Biophysical models can be used to predict the sequence of electrical heart activation in a patient given a set of parameters which should be personalized to the patient. In this paper, we use electroanatomical maps to personalize the endocardial activation of the right ventricle, and the different tissue conductivities in a pig model with left bundle branch block, to reproduce personalized biventricular activations. Following, we tested the personalized heart model by virtually simulating cardiac resynchronization therapy.

Keywords Cardiac resynchronization therapy - Tissue properties personalization - Biophysical modeling 


\title{
Prediction of CRT Activation Sequence by Personalization of Biventricular Models from Electroanatomical Maps
}

\author{
Juan Francisco Gomez ${ }^{1,3}$, Beatriz Trenor ${ }^{1}$, and Rafael Sebastian ${ }^{2(\bowtie)}$ \\ 1 Centre for Research and Innovation in Bioengineering (Ci2B), \\ Universitat Politecnica de Valencia, Valencia, Spain \\ 2 Computational Multiscale Simulation Lab (CoMMLab), \\ Department of Computer Science, Universitat de Valencia, Valencia, Spain \\ rafael.sebastian@uv.es \\ 3 International University of Valencia (VIU), Valencia, Spain
}

\begin{abstract}
Optimization of lead placement and interventricular delay settings in patients under cardiac resynchronization therapy is a complex task that might benefit from prior information based on models. Biophysical models can be used to predict the sequence of electrical heart activation in a patient given a set of parameters which should be personalized to the patient. In this paper, we use electroanatomical maps to personalize the endocardial activation of the right ventricle, and the different tissue conductivities in a pig model with left bundle branch block, to reproduce personalized biventricular activations. Following, we tested the personalized heart model by virtually simulating cardiac resynchronization therapy.
\end{abstract}

Keywords: Cardiac resynchronization therapy • Tissue properties personalization · Biophysical modeling

\section{Introduction}

Patients with a complete left bundle branch block (LBBB), show a significant delay between activation of the interventricular septum and activation of the left ventricular (LV) free wall. Therefore, decreasing the delay by pacing may restore mechanical contraction. Cardiac Resynchronization Therapy (CRT) is a successful electrical treatment for patients with ventricular dyssynchrony. During CRT, two synchronized electrical stimuli are usually delivered to reduce ventricular dyssynchrony. One stimulation lead is usually placed on the apex of the right ventricle (RV), and the other one on the epicardium of the LV lateral wall. Large randomized clinical trials $[2,8]$ have led to the widespread adoption of CRT in patients with a prolonged QRS duration $\geq 120 \mathrm{~ms}$. A significant intraventricular conduction delay reflected by a prolonged QRS duration $(\geq 150 \mathrm{~ms})$ with LBBB morphology remained the main indication to CRT. Nevertheless, a significant 
proportion of implanted patients fails to respond sufficiently or in a predictable manner. There are a number of critical factors that have to be considered for CRT to be effective.

Since ECG criteria may be imperfect, there is increasing interest in advanced multimodality imaging to improve patients selection, guide LV catheter delivery and identify patients at risk for poor outcomes and serious ventricular arrhythmias [4].

Computational models for biophysical simulation are valuable tools for better understanding pacing-based therapies such as CRT [7], providing additional information to the clinician on its optimal parameters for a given patient $[3,10]$. In this study, we show a pipeline to personalize a computational model of a pig heart, using electro-anatomical maps (EAMs) acquired in LBBB, which is subsequently used to predict the sequence of activation under CRT.

\section{Materials and Methods}

\subsection{Construction of Anatomical Models}

For this study, we used two datasets from the CRT-EPiggy19 Challenge publicly available. The anatomical models used were segmented in a previous work by Soto-Iglesias et al. [9], and were afterwards improved by including a more realistic fiber orientation description [5]. The first dataset corresponded to a pig heart labeled as Neus, a non-infarcted case with a LBBB activation pattern, which was considered a CRT non-responder. The second model, labeled as Kira, was an infarcted case with a large scar located in the antero-septal and septo-apical areas, and a clear LBBB pattern and electrical dyssynchrony, who was considered a CRT responder.

The biophysical finite element models provided for each case were enhanced to obtain the required properties for simulation. First, models were remeshed with hexahedra to meet the requirements of our biophysical solver (ELVIRA) and to reduce the degrees of freedom. Second, all the properties of the original model were transfer to the new volumetric model and extra information related to endocardial, mid-myocardial and endocardial regions were added. For each case, we calculated the conduction velocities from the mesh at each segment of the AHA, for both the endocardium and the epicardium (we divided each AHA segment in two subregions). We observed high conduction velocities in the endocardium and the LV lateral wall at basal areas. We assumed that the effect was due to the Purkinje system, which was functional and allowed retrograde activation. Figure 1 shows the clear effect of the fast endocardial layer in the EAM of the case Neus in LBBB, where the isochrones are much wider (faster conduction velocity) in the LV endocardium and the LV epicardium. For instance in the models Kira and Neus, the average conduction velocities measured in all the endocardial AHA segments were $1.29 \pm 0.69 \mathrm{~m} / \mathrm{s}$ and $1.52 \pm 0.97 \mathrm{~m} / \mathrm{s}$, respectively. Therefore, we added a fast endocardial conduction layer that was one element thick, to the RV and LV. We are aware that pig hearts present a 


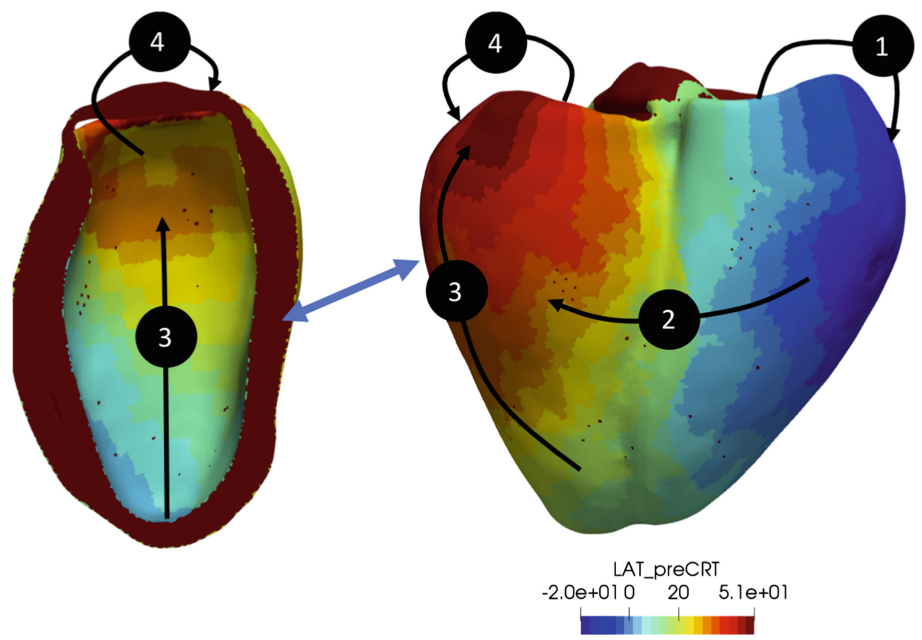

Fig. 1. Analysis of electrical propagation in ground-truth data. LBBB cases show a sequence of activation that can be summarized in four steps: (1) activation from RV endocardium tu RB epicardium and septal wall, (2) transmural propagation from RV to LV, (3) propagation from LV apex to base, with fast activation of the endocardium and slow in the epicardium, (4) transmural propagation from fast endocardium to epicardium in the LV lateral wall.

transmural Purkinje system, but since we do not have additional data, we opted only for the fast endocardial layer.

\subsection{Biophysical Simulation}

Detailed multiscale simulations were carried out for each ventricular model. Cellular electrophysiology was simulated by the ten Tusscher model considering transmural cellular heterogeneity, and electrical propagation by monodomain model.

\subsection{Personalization of LBBB Activation Sequence}

In order to obtain a personalized activation sequence for the LBBB patterns we developed the following methodology, summarized in Fig. 2. First, from the EAM, the LAT maps for the RV epicardium and the septal region of the LV endocardium are selected. In LBBB, those regions are expected to be activated by the RV endocardial sequence, since they are the closest ones. From the selected regions an inverse propagation to the RV endocardium is performed to obtain the original pattern of activation and the activation times in the RV endocardium. Once the RV stimulation sequence is obtained, it is used to activate the RV, obtaining the expected LBBB pattern. Following, the simulated LAT map is compared to the EAM to adjust the longitudinal and traversal conductivities. 
Since the model includes a fast endocardial layer that functionally mimic the Purkinje system, those conductivities have to be set. Myocardial conductivities are obtained by looking at the epicardium isochrones, while Purkinje ones are derived from the endocardium. In models including scar, the elements were properly labeled, and the conductivity was set as $25 \%$ of the normal myocardium.

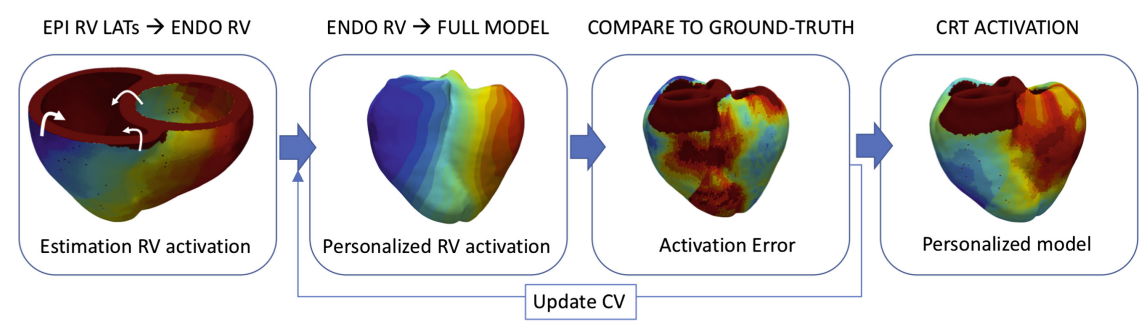

Fig. 2. Pipeline for personalization of the model parameters and simulation of CRT. $\mathrm{EPI}=$ Epicardium $; \mathrm{ENDO}=$ Endocardium

\section{Results}

\subsection{LBBB Activation Sequences}

For each of the models we followed the methodology developed to obtain the activation sequence of the RV endocardium. Conductivities were optimized in all the models iteratively by performing simulations and comparing the differences with the EAM data. Final conduction velocities are summarized in Table 1, together with mean square errors and total activation times. Note that we aim to reduce the average difference between LAT maps and not the final total activation time (TAT).

Figure 3 shows the results for the non-ischemic case Neus. As can be observed for the model Neus, the activation sequence is very similar at both the endocardium and the epicardium. Since the EAMs do not include the endocardium of the RV, the colormaps have been adjusted to be comparable, using the same scale, by shifting to the initial times (depolarization of the RV epicardium). The effect of the fast activated layer at the endocardium was key to obtain similar maps at the LV lateral wall. The TATs match between EAM and simulation, which is $71 \mathrm{~ms}$. In the simulations, the isochrones are smoother, and do not reproduce the changes in the depolarization wavefront curvature observed in EAMs (Fig. 3(a) and (c)), which are probably due to the sampling and interpolation of the data. Even with the fast activation layer, the endocardium in the EAM is slightly faster (wider isochrones), than the simulation (Fig. 3(b) and (d)).

For the infarcted case, the model Kira, we simulated the activation sequences by personalizing the model as in Neus model. The real shape of the scar was not provided, but only which AHA regions were affected, and therefore, we simply 
reduced $25 \%$ the conduction velocities on those regions. As can be observed in Fig. 4(a), the epicardial RV activation was well reproduced, introducing the fast endocardial layer in the RV. However, we observed in the EAM a very slow conduction velocity (CV) in some regions of the LV endocardium (Fig. 4(c) top), compared to the LV epicardium (Fig. 4(c) down). That was unexpected since the scar in Kira model extends to the epicardium, but not the endocardium, and therefore we expected exactly the opposite result, which matches simulations (results not shown). Therefore, we updated the model to extend the scar to the endocardium. With the updated conductivities, we simulated properly the isochrones in LBBB in Kira model, with exception of the epicardium of the anterior wall and the apical region of the lateral wall Fig. 4(b) and (d). Those regions showed an abnormal fast CV $(2,062,29 \mathrm{~m} / \mathrm{s})$ compared to the rest of the model (Fig. 4(b) top vs down), which was very remarkable considering that there is a large epicardial scar right under the anterior wall. The fast epicardial CV in the anterior versus posterior wall was present in both Kira and Neus models, although in the last was less marked.

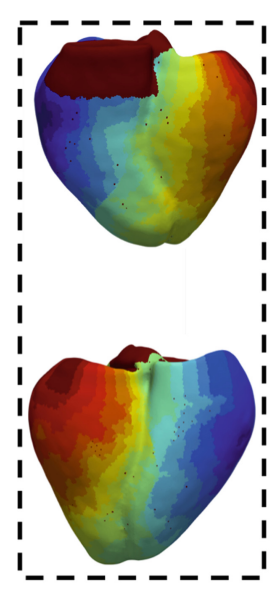

(a)

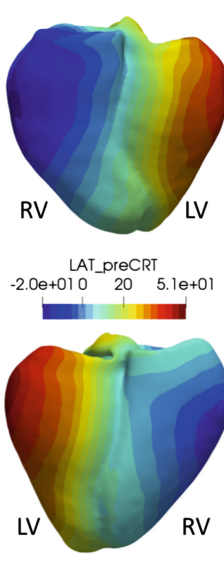

(b)

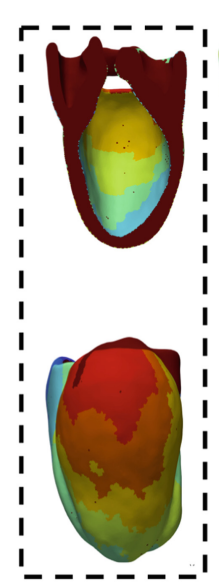

(c)

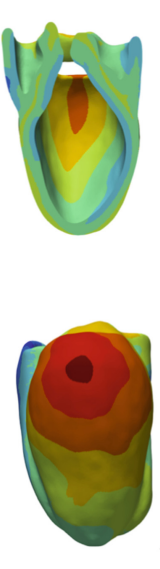

(d)

Fig. 3. Personalized LBBB sequence of activation for model Neus. (a) and (c) are anterior an posterior views of the model showing local activation times in the epicardium of the model (left) and the ground-truth data (right). (b) and (d) show endocardial and epicardial views of the LV lateral wall, respectively, where left subfigures correspond to simulations and right subfigures to ground-truth.

\subsection{CRT Activation Sequences}

Once the conductivities were estimated for the LBBB sequences, they were used for the CRT simulations. A priori, the fast endocardial layer was kept as it was functional for the LBBB sequences, and was expected to activate as well from 


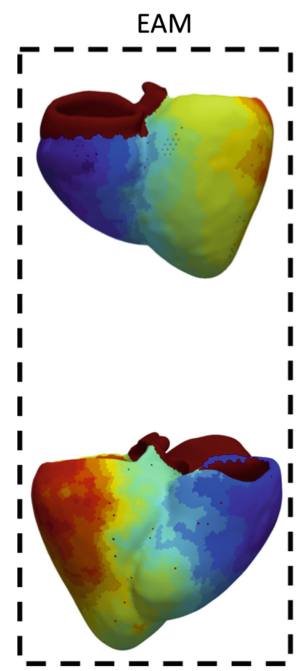

(a)

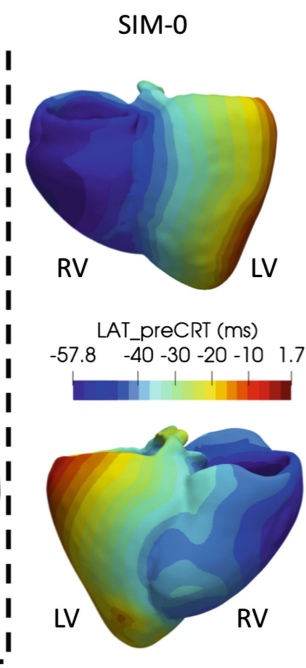

(b)

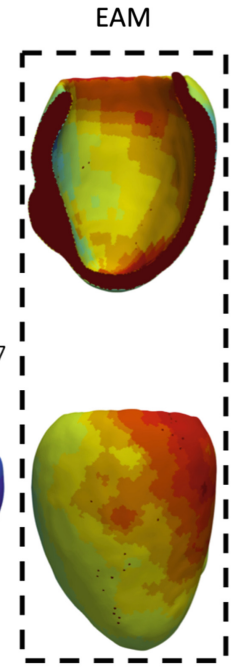

(c)
SIM-0
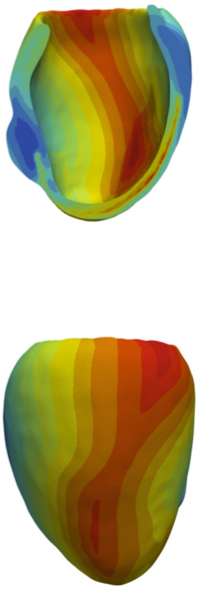

(d)

Fig. 4. Personalized LBBB sequence of activation for model Kira. (a) and (c) are anterior an posterior views of the model showing local activation times in the epicardium of the model (left) and the ground-truth data (right). (b) and (d) show endocardial and epicardial views of the LV lateral wall, respectively, where left subfigures correspond to simulations and right subfigures to ground-truth.

the CRT leads, or remote depolarization wavefronts travelling across the ventricles. Note that the fast endocardial layer can be personalized independently for the RV and LV. In the model Neus (see Fig. 5) the CRT leads were placed in the earliest activation sites, which were the RV endocardium (mirroring the earliest epicardial activation) and the apex of the LV epicardium (see Fig. 5(b)). The activation of the RV epicardium in the EAM was finished in less than $30 \mathrm{~ms}$, which could be only explained if a fast RV endocardial layer spreads quickly the initial CRT lead impulse over the endocardium. After $10 \mathrm{~ms}$ of the RV epicardial breackthrough the wavefront reached the LV endocardium at the lower-septum (see Fig. 5(e)), coinciding with the activation of the LV lead, which was probably set $20 \mathrm{~ms}$ after the RV lead. On the LV endocardium the depolarization wavefront advanced slower than in the LBBB scenario, showing a much slower $\mathrm{CV}$, or a poor access to the Purkinje system (see Fig. 5(d)). The simulation, reproduced well the activation pattern of the RV epicardium, with some differences at the basal region, and also the anterior and posterior walls. At the LV endocardium, in contrast to the EAM, the model showed much faster conduction velocities than the EAMs, and an activation sequence from endocardium to epicardium in the LV lateral wall due to the fast layer (see Fig. 5(e)). That was the largest difference, since in the EAM the activation: (i) follow a epicardium to endocardium activation sequence, with a large delay transmurally; and (ii) early activated regions in the base of the lateral wall, which coincided with the latest 
Table 1. Estimation of CV parameters for computational models. CV = Conduction velocity (longitudinal/transmural); SIM = simulation;

\begin{tabular}{l|l|l|l|l|l|l|l}
\hline \multicolumn{2}{l|}{ Model } & \multicolumn{2}{l|}{ Tissue $(\mathrm{m} / \mathrm{s})$} & \multicolumn{2}{l|}{ PKN $(\mathrm{m} / \mathrm{s})$} & \multicolumn{2}{l}{ TAT $(\mathrm{ms})$} \\
\hline Name & Seq & $C V_{L}$ & $C V_{T}$ & $C V_{L}$ & $C V_{T}$ & SIM & EAM \\
\hline Neus & LBBB & 0.5 & 0.25 & 2.6 & 0.54 & 71 & 71 \\
\hline Kira & LBBB & 1.78 & 0.58 & 1.4 & 1.3 & 68 & 59.5 \\
\hline Neus & CRT & 0.9 & 0.5 & 1.0 & 0.9 & 65 & 58 \\
\hline Kira & CRT & 1.78 & 0.58 & 1.4 & 1.3 & 49 & 40 \\
\hline
\end{tabular}

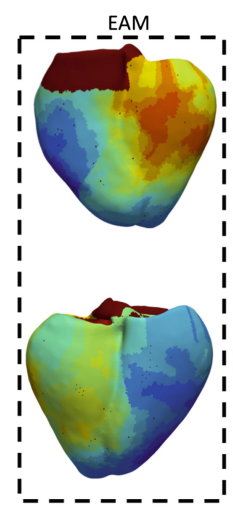

(a)

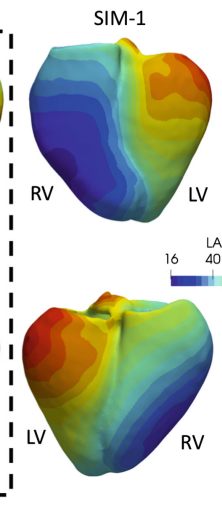

(b)

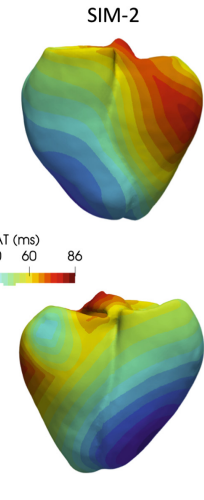

(c)

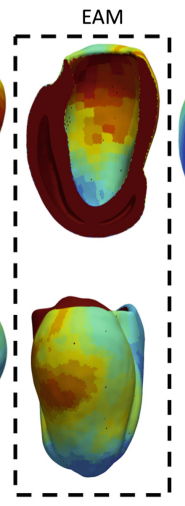

(d)

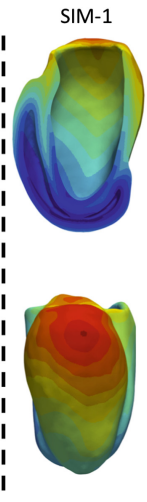

(e)

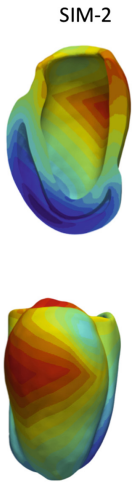

(f)

Fig. 5. Personalized simulations for prediction of CRT sequence of activation in Neus Model. (a) and (d) show anterior and posterior views of the EAM, and endocardial and epicardial views of the LV lateral wall, respectively. (b) and (e) show the simulations results using the personalized values obtained from the LBBB model. (c) and (f) show the simulations using personalized values obtained from the data post CRT implantation.

activated region in LBBB. Therefore, the model could not reproduce properly the activation sequence in the LV, which is very difficult to explain unless the LV lead would have been placed in the latest activated region in LBBB, that is the basal region of the LV lateral wall. Therefore, we updated the CRT lead locations to have a lead in the RV endocardium, and a lead in the LV epicardium lateral wall. In addition, we adjusted the fast conduction layer of the LV to an intermediate $\mathrm{CV}$ between Purkinje and myocardium. The results improved significantly (see Fig. 5(c) and (f), and the sequence of activation matched properly between simulations and EAMs, i.e., there was a epicardial to endocardial activation sequence in the $L V$, and a delayed activation of the basal region of the LV endocardium.

In the case of Kira model, using the same set of conductivities obtained from the LBBB model did not obtain good agreement between EAM and simulations. 


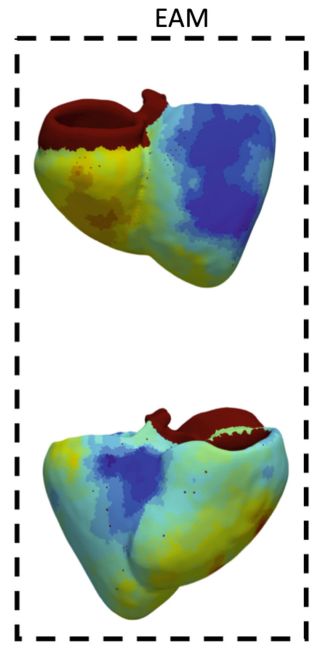

(a)

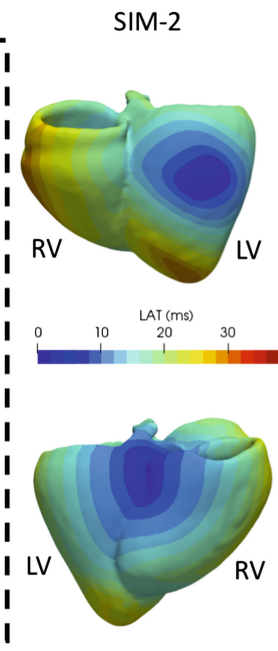

(b)

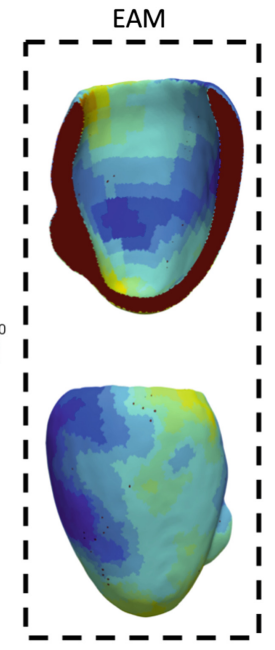

(c)
SIM-2

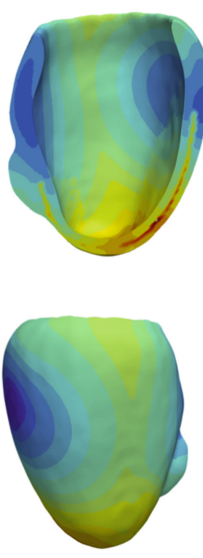

(d)

Fig. 6. Personalized simulations for prediction of CRT sequence of activation in Kira Model. (a) and (c) show anterior and posterior views of the EAM, and endocardial and epicardial views of the LV lateral wall, respectively. (b) and (d) show the simulations using personalized values obtained from the data post CRT implantation.

Kira model activates completely in $35 \mathrm{~ms}$, which can only be accomplished if the conduction velocities all over the model are really high. If one considers a transmural Purkinje system, with a very fast access from the lead, such CVs could have been obtained. Average conduction velocities all over the model were $2.22 \pm 1.17 \mathrm{~m} / \mathrm{s}$. Figure 6 shows the isochrones of Kira model obtained from EAM and from simulations. After recalculating all the conductivities, not based on LBBB but on CRT EAM, we could obtain similar results except in the anterior wall that showed a very large initial activated area.

\section{Discussion and Conclusions}

The accurate reproduction of activation patterns from real patient data using computational models is a complex task due to the large number of unknowns, variability and heterogeneity in the heart. Comparing simulations with EAMs introduces additional errors since the mapping is done sequentially and the activation of the heart may vary from beat to beat. In addition, it is very tricky to annotate the local activation times and hence to have a meaningful and faithful activation sequence of the patient that is spatio-temporally coherent. Therefore, differences are expected a priori, sometimes large, between simulations and EAMs.

An important feature added to our pipeline to obtain more accurate results was the fast conduction endocardial layer, which was independent for LV and 
RV. Since the LBBB was artificially induced by blocking the LBB at the His Bundle level, the remaining structure of the Purkinje system was still functional, and could be activated retrogradely. This hypothesis was clearly confirmed in cases such as Neus, where the wavefront coming from the RV speeds up in the LV endocardium, advancing the LV epicardial wavefront and producing much wider isochrones. In addition, once the effect of the fast endocardium reaches the epicardium transmurally, it can be noticed a fast apex to base activation in the LV lateral wall, see Fig. 1. All these observations were properly reproduced once the fast conduction layer was included in the LBBB computational model. In the original paper by Soto-Iglesias et al. [9], authors reported that in the Neus model the fast conduction by Purkinje was not active in LBBB or under CRT, which does not agree with our observations, where this effect in LBBB is very clear (see Fig. 1), and was validated by the model who reproduced much more accurately the EAMs once the layer was included.

Under CRT in Neus model, the late activated basal regions of the LV initially differed from that of the simulations. Analysing the EAMs under CRT, it could be observed that the LATs at the LV basal region show large gradients transmurally, i.e., the epicardium was activated around $40 \mathrm{~ms}$ earlier than the corresponding endocardium. That effect was not observed during LBBB. We performed simulations with and without a fast endocardial conduction layer on the LV, and we concluded that there was a fast endocardial layer in the LV, but it was slower than in the case of LBBB, probably due to a different access of the Purkinje system. The activation of the LV epicardium under CRT was surprisingly high, almost as fast as the fast endocardial layer, which could be only explained by the presence of the Purkinje system in the epicardium of pig hearts, where the system if fully transmural [6]. The effect was also observed in Kira model, and remarkably in both cases the anterior wall showed much higher CVs than the posterior. We hypothesized that the anterior branch of the Purkinje system could have been functional whereas the posterior branch might be damaged. Auricchio et al. [1], found using concact and non-contact mapping that around $32 \%$ of $\mathrm{LBBB}$ patients, had $\geq 20 \mathrm{~ms}$ between the beginning of activation in the RV endocardium versus the LV endocardium, which was considered as incomplete LBBB. Complete LBBB patients show interventricular delays $>40 \mathrm{~ms}$. We consider that this could also be a case of incomplete LBBB, since the delay between the RV breackthroughs and the LV breacktrough was around $20 \mathrm{~ms}$. In addition, as described in [6] for pig hearts the density of PMJs in the epicardium is larger and particularly in the anterior region compared to the posterior.

In conclusion, we have developed a pipeline to personalize globally, computational models of the heart from EAMs, to predict the electrical activation sequence of a given patient under CRT. Results show that a much detailed personalization of $\mathrm{CVs}$ is required to reproduce properly the activation sequence. 


\section{References}

1. Auricchio, A., et al.: Characterization of left ventricular activation in patients with heart failure and left bundle-branch block. Circulation 109(9), 1133-9 (2004). https://doi.org/10.1161/01.CIR.0000118502.91105.F6

2. Bristow, M.R., et al.: Comparison of medical therapy, pacing, and defibrillation in heart failure (COMPANION) investigators: cardiac-resynchronization therapy with or without an implantable defibrillator in advanced chronic heart failure. N. Engl. J. Med. 350(21), 2140-50 (2004). https://doi.org/10.1056/NEJMoa032423

3. Carpio, E.F., et al.: Optimization of lead placement in the right ventricle during cardiac resynchronization therapy. A simulation study. Front. Physiol. 10, 74 (2019). https://doi.org/10.3389/fphys.2019.00074

4. Dawoud, F., et al.: Non-invasive electromechanical activation imaging as a tool to study left ventricular dyssynchronous patients: implication for crt therapy. J. Electrocardiol. 49(3), 375-82 (2016). https://doi.org/10.1016/j.jelectrocard.2016. 02.011

5. Doste, R., et al.: A rule-based method to model myocardial fiber orientation in cardiac biventricular geometries with outflow tracts. Int. J. Numer. Method Biomed. Eng. 35(4), e3185 (2019). https://doi.org/10.1002/cnm.3185

6. Garcia-Bustos, V., Sebastian, R., Izquierdo, M., Molina, P., Chorro, F.J., RuizSauri, A.: A quantitative structural and morphometric analysis of the purkinje network and the purkinje-myocardial junctions in pig hearts. J. Anat. 230(5), 664-678 (2017). https://doi.org/10.1111/joa.12594

7. Lopez-Perez, A., Sebastian, R., Ferrero, J.M.: Three-dimensional cardiac computational modelling: methods, features and applications. Biomed. Eng. Online 14, 35 (2015). https://doi.org/10.1186/s12938-015-0033-5

8. Moss, A.J., et al.: MADIT-CRT trial investigators:cardiac-resynchronization therapy for the prevention of heart-failure events. N. Engl. J. Med. 361(14), 1329-38 (2009). https://doi.org/10.1056/NEJMoa0906431

9. Soto Iglesias, D., et al.: Quantitative analysis of electro-anatomical maps: application to an experimental model of left bundle branch block/cardiac resynchronization therapy. IEEE J. Transl. Eng. Health Med. 5, 1900215 (2017). https://doi. org/10.1109/JTEHM.2016.2634006

10. Tobon-Gomez, C., et al.: Understanding the mechanisms amenable to crt response: from pre-operative multimodal image data to patient-specific computational models. Med. Biol. Eng. Comput. 51(11), 1235-50 (2013). https://doi.org/10.1007/ s11517-013-1044-7 


\section{Author Queries}

Chapter 36

\begin{tabular}{|c|l|l|}
\hline $\begin{array}{l}\text { Query } \\
\text { Refs. }\end{array}$ & Details Required & $\begin{array}{l}\text { Author's } \\
\text { response }\end{array}$ \\
\hline AQ1 & $\begin{array}{l}\text { Per the Table of Contents, we have expanded the author } \\
\text { name in the author group. Please check and confirm if } \\
\text { this is okay. }\end{array}$ & $\begin{array}{c}\text { The opening quotes does not have a corresponding clos- } \\
\text { ing quotes in the sentence 'The results improved signif- } \\
\text { icantly...' Please insert the quotes in the appropriate } \\
\text { position. }\end{array}$ \\
\hline AQ2
\end{tabular}

